\title{
RESPUESTA A LOS ATENTADOS DE BARCELONA
}

\author{
Martí Boneta I Carerra ${ }^{1}$
}

\section{Resumen:}

El terrorismo yihadista es otra vez noticia, ya que la semana pasada se produjeron unos nuevos atentados, esta vez en Cataluña. Las primeras horas después de este suceso hay que atender lo mejor posible a las víctimas y familiares y condenar estos hechos. También hay que dejar que la justicia y la policía y la justicia hagan su trabajo. En el artículo se analizan algunas de las causas de estos hechos, se plantean las preguntas de las razones del porqué unos jóvenes se entreguen al terror y cuales son las pasiones y las circunstancias que los llevan a cometer actos tan horribles por una causa tan espantosa. En estas últimas décadas, Cataluña ha experimentado un gran incremento de población, procedente de muchos países, ya se puede hablar que somos una sociedad multicultural. La política educativa ha sido clave para la normalización de este proceso.Tres elementos son claves para la solución de este terrorismo: personas, problema y proceso. Separar estos tres elementos nos permitirá ser sensibles y comprensivos con las personas, duros con el problema y equitativos en el proceso.

Palabras claves: personas, problema y proceso

\section{RESPOSTA AOS ATENTADOS DE BARCELONA}

\section{Resumo:}

O terrorismo jihadista é outra vez noticia, já que na semana passada efetuou um novo atentado, desta vez na Catalunha. Nas primeiras horas depois do sucedido há que socorrer o melhor possível as vítimas e familiares e condenar estes atos. Também há que deixar que a justiça e a polícia façam o seu trabalho. Neste artigo analisam-se algumas das causas destes atos, levantam-se questões sobre as razões que levam jovens a se entregar ao terrorismo e quais as paixões e circunstâncias que os levam a cometer atos tão horrendos por uma causa tão assustadora. Nestas últimas décadas, a Catalunha assistiu a um grande aumento da sua população, chegaram pessoas provenientes de muitos países, e já se pode dizer que somos uma sociedade multicultural. A política educativa tem sido fundamental para este processo de integração.Três elementos são indispensáveis para solucionar o terrorismo: pessoas, problema e processo. A separação destes três elementos nos permitirá ser sensíveis com as pessoas, duros com o problema e equitativos com o processo.

Palavras chaves: pessoas, problema e processo.

\footnotetext{
${ }^{1}$ Consejería de Educación de la Generalitat - Ministério de Educação da Espanha e Banco Popular OIKOCREDIT da Espanha. E-mail: mboneta@xtec.cat.
} 


\title{
ANSWER TO THE ATTACKS OF BARCELONA
}

\begin{abstract}
:
Jihadist terrorism is on the news once again, since last week it carried out a new attack, this time in Catalonia. In the first hours after the event, must be given to the victims and their families the best help and condemn these acts. It should also be left to justice and police to do their job. In this article are analyzed some of the causes of these acts, questions are raised about the reasons why young people are involved in terrorism and what passions and circumstances lead them to commit such horrendous acts for a such scary cause. In last decades, Catalonia has witnessed a large increase in its population, people from many countries have arrived and we can already talk about a multicultural society. An educational policy has been central to the integration process. Three elements are indispensable to solve terrorism: people, problem and process. The separation of these three elements will allow us to be sensible to people, tough on the problem and equitable with the process.
\end{abstract}

Keywords: people, problem and process.

El pasado jueves 17 de agosto se han producido unos graves atentados en pleno centro de Barcelona, en la famosa y concurrida Ramblas, y en Cambrils. En primer lugar, en estas primeras horas hay que dejar que la policía haga su trabajo de detención de los culpables y que los lleve a la justicia. Como hemos podido ver la actuación de los Mossos d'Esquadra, policía de Cataluña, y demás fuerza seguridad del Estado han hecho un gran trabajo. En este primer momento, también hay que socorrer a las víctimas, a los heridos, darles la mayor atención satinaría posible. También acompañar física y psicológicamente a los familiares y de sus amistades de las víctimas mortales. Seguro que ninguna palabra de consuelo les devolverá sus seres más queridos, pero hemos de estar a su lado. Acompañarles en su dolor, tanto a nivel de ayuda profesional como de la solidaridad de toda la sociedad, e intentar que puedan llevarse hacia sus lugares de origen a los cuerpos de los fallecidos. También hay que decir que la presencia y el trabajo de las diferentes autoridades han estado a su altura. Poniéndose, y como debe de ser, al servicio de las víctimas, en particular, y de toda la sociedad, en general.

El minuto de silencio del día después en Plaza Catalunya, con el final de unos grandes aplausos y el lema "No es fan por" son los signos evidentes de una sociedad madura y que actos como este no les harán cambiar su ritmo normal de actividad y de convivencia.

Una vez resueltos estos temas de los primeros días, habrá que analizar muy profundamente las causas y buscar respuestas a estas y otras preguntas: ¿por qué hay jóvenes entregados al terror? ¿qué hay en sus cabezas? ¿cuáles son las razones y las pasiones y las 
circunstancias que los llevan a cometer actos tan horribles por una causa tan espantosa? Las respuestas a estas preguntas aportarán las ideas y los medios para poder evitar un nuevo horror.

En un primer momento es necesario solucionar lo inmediato. Pasa lo mismo que cuando hay un incendio, primero tienen que actuar los bomberos para apagar los más rápidamente posible las llamas que destruyen un espacio y socorrer a las personas que están en el lugar. Después con más calma se analizará la causa y las causas para intentar que no vuelva a ocurrir nunca más. Las dos cosas no se pueden hacer a la vez, hay un tiempo y unas necesidades en cada momento.

En el caso del atentado terrorista de Barcelona, las causas son múltiples y, creo, que nos equivocaríamos si las reducimos por el momento que Cataluña está pasando, el llamado procès, o por el tema del gran auge del turismo en Barcelona. Por cierto que algunos le han querido denominar turismofobia, aquí no es el espacio de analizar este tema, pero sólo quiero destacar que todas las fobias son negativas y que si queremos analizar las grandes cifras económicas, el turismo supone el 12\% del PIB de la ciudad de Barcelona, y que da muchos puestos de trabajo (aquí también entraría la reflexión y de una amplio análisis de la calidad de estos trabajos, sólo apunto el movimiento tan interesante de las Kelys). Seguro que se podría administrar de forma diferente y que tiene algunas consecuencias muy negativas como es la ocupación de unos espacios y de unas viviendas que expulsan a sus habitantes de los lugares que han vivido durante muchos años por el aumento de los precios de los pisos y apartamentos. Pero el tema es mucho más amplio e internacional, no sólo le ocurre a Barcelona, y además tendríamos que cuestionarnos el bajo ritmo de construcción de vivienda social realizado por las diferentes administraciones durante estos años. Además si la vivienda es un derecho, recogido por los diferentes tratados internacionales y nacionales, nos tendríamos que preguntar si debe estar sujeta a los flujos de mercado y de la especulación financiera.

También se podría culpabilizar a que Catalunya cuenta con mucha emigración. La necesidad de cambio de residencia es un derecho inalienable garantizado en la Declaración Universal de los Derechos Humanos, en su artículo 13:

toda persona tiene derecho a circular libremente y a elegir su residencia en el territorio de un Estado. Toda persona tiene derecho a salir de cualquier país, incluso el propio, y a regresar a su país. 
Ese esencial derecho a emigrar, a movilizarse, (sólo cercenado en los regímenes políticos autoritarios y dictatoriales) se complementa con otro fundamental que busca la protección del ser humano en condiciones de vulnerabilidad y persecución: el derecho al refugio y al asilo, recogido también en la carta fundamental de los derechos del hombre. En la Declaración Universal de los Derechos Humanos, artículo 14, se puede leer: "En caso de persecución, toda persona tiene derecho a buscar asilo, y a disfrutar de él, en cualquier país".

Como decía, las causas de estos terribles atentados son muy amplias en el tiempo y en el espacio. También recordar, que las principales víctimas en el mundo del terrorismo de ISIS son los mismos musulmanes ya que más del $95 \%$ de las muertes causadas por ellos tienen lugar en sus países y son de la misma religión. Y aquí quiero ya apuntar un tema fundamental que desde el primer momento hay que decirlo. La inmensa mayoría de las personas de religión musulmana, quieren vivir en paz y serían incapaces de matar a nadie. Es triste, pero siempre que hay un atentado de este tipo en Occidente, algunas personas quieren culpabilizar a toda una comunidad. Esta, y en Barcelona también ha ocurrido, tiene que salir enseguida a los medios de comunicación lamentando los hechos (ya sean entidades sociales o religiosas) y que no culpabilicen a todos ellos. También hay conceptos muy equivocados, que a veces llevados por la ignorancia u otras sabiendo que vas hacer un mal, en identificar musulmán con árabe. Y eso no es así. El país del mundo donde hay más musulmanes es Indonesia. Tampoco el identificar el terrorismo de ISIS con todos les musulmanes. Es como dijéramos que la Extrema Derecha, y este es un fenómeno también muy grave en diferentes países occidentales por la gran expansión que ha tenido lugar estos años y por los resultados electorales que tiene por el apoyo de una parte de la población, representa a la Derecha política. No tiene nada que ver el partido Republicano de Francia con el Frente Nacional. Ni Marie Le Pen, con Teresa May o Ángela Merkel.

Si analizamos las causas en el espacio y tiempo, no nos podemos olvidar de la invasión de Iraq, que lejos de resolver un problema lo agrandó, o bien la situación de Palestina. Sólo para poner dos ejemplos de que ni la Comunidad Internacional en su conjunto, ni Naciones Unidas ni los principales Estados han resulto.

A nivel de Cataluña, hay que decir que en diez años, entre finales de los años 90 del siglo XX y la primera década del XXI, la población creció en un 20\%, pasando de 6 millones de habitantes a 7,2 millones. En un espacio, más bien reducido: Cataluña tiene 30 mil kilómetros cuadrados. Prácticamente todas las comarcas, no sólo las del área industrial de 
Barcelona, han tenido la llegada masiva de persona de otros países. Esto fue por la gran demanda de puestos de trabajo que la población autóctona no podía o, en algunos casos, no quería asumir: construcción, agricultura, labores domésticas, cuidados de las personas mayores... Sin esta llegada de personas, habría sido imposible el crecimiento económico. La crisis del 2010 frenó este proceso, y en algunos casos algunas personas regresaron en sus lugares de origen al no quedarse sin trabajo aquí. Los centros educativos acogieron a toda esta población en edad escolar.

Esta llegada de población hizo que se incrementasen notablemente el número de plazas escolares ofertadas hasta ese momento. En España y en Cataluña es obligatoria la escolarización de todos los niños, niñas y jóvenes entre 6 y 16 años. La realidad, es que de 3 a 6 también están escolarizados casi el $100 \%$ de la población y una mayoría de los de 16 a 18 años. Cabe decir que nos costó muchos años este objetivo de niveles de escolarización, de hecho este $100 \%$ se consiguió a partir de la LOGSE, ley de educación que se aprobó a medianos de los años 90 del siglo pasada. Este ha sido un gran reto conseguido, y que ya a principios del siglo XX era un objetivo de movimientos pedagógicos, políticos y sociales. Como ha costado tanto, no queremos renunciar y además, en estos momentos, hay un gran movimiento de renovación pedagógica que no sólo quiere mantener esta escolarización en términos cuantitativos, si no también cualitativos.

Cabe decir, que el gobierno de la Generalitat, tomó la valiente y decisiva decisión de hacer un sistema educativo integrador e inclusivo. No se harían escuelas exclusivas para emigrantes, separados de la otra población. Se hizo un gran esfuerzo económico y profesional en que se ampliaban las escuelas existentes y si por razones de demanda se abrían de nuevas estas eran para toda la población. Desde la Consejería de Educación de la Generalitat de Catalunya, se puso en marcha el Pla LIC, (Lengua, Interculturalidad y Cohesión Social). Con este Plan se abrieron dentro de las mismas escuelas, muchas Aulas de Acogida con el incremento de plantilla de profesorado y de recursos materiales en los mismos centros. Estas aulas tenían un doble objetivo: acelerar el procesos de aprendizaje del catalán, (lengua de aprendizaje e institucional del centro) y realizarles una buena acogida y acompañamiento. Los alumnos pasaban a partir de los 8 años un máximo de 10 horas semanales para que pudieran acelerar el proceso de aprendizaje del catalán el más rápido posible y que se sintieran el máximo de acogidos, acompañándoles en este proceso de adaptación de la nueva sociedad. Pero lo importante es que la mayoría de su jornada escolar, estos alumnos estaban en sus 
aulas, con el resto de compañeros y compañeras. No se separaban en centros. El profesorado hizo un gran esfuerzo de adaptación de esta nueva realidad. También se llevaron a cabo los Planes Educativos de Entorno, convenios con diferentes ayuntamientos para intentar que en el tiempo de tiempo libre estos alumnos participasen de actividades extraescolares y sociales. El resultado de este proceso vivido en tan pocos años ha sido que los centros educativos hay una gran variedad de lenguas, más de 150, culturas, religiones, razas... Personalmente creo que es una gran riqueza para todos y todas.

Por otra parte, la Secretaria d'Igualtat, Migracions i Ciutadania de la Generalitat, Gobierno de Cataluña impulsa diferentes planes y programas para poder aplicar y desarrollar las políticas de igualdad y migraciones, algunos de ellos conjuntamente con la administración local, como la acogida y soporte a las personas migradas.

El Consorci d'Educació de Barcelona, responsables de la gestión de los Centros Educativos de la ciudad de Barcelona, tiene previsto iniciar el curso que viene desarrollar un plan para que la Ciudadanía y los Valores, estén mucho más presentes en la vida de los centros escolares. Que los estudiantes, en todos los niveles educativos, tomen conciencia de la importancia de vivir en una sociedad del siglo XXI. Fomentar proyectos que en las diferentes etapas educativas, los alumnos tengan la posibilidad de desarrollar el altruismo y la cooperación, comprometerse con su comunidad. Es una respuesta clara y decisiva a los hechos ocurridos.

¿Todo ha salido bien? Está claro que no. Se ha dicho que el profesorado ha hecho y está haciendo un gran proceso de reconversión profesional, pero con una plantilla de más de 120.000 profesionales, seríamos ingenuos pensar que todos están por la labor. Ha fallado el sistema de reparto de alumnos. Podemos tener centros con el $80 \%$ de alumnos emigrantes y otros sólo con un 5\%. El tema no es sólo de adaptación cultural, también hay temas económicos muy difíciles de resolver. La crisis económica ha afectado a una gran parte de la sociedad, pero ha sido muy importante en sectores de las personas que vinieron de fuera. La construcción, que daba muchos puestos de trabajo, disminuyó mucho en un espacio de tiempo muy corto, dejando un número muy importante de personas al paro. Muchos de los que se quedaron sin trabajo fueron estos nuevos habitantes. Los resultados de la obtención del Graduado Escolar a final de la escolarización obligatoria a los 16 años es muy dispar. Si los alumnos procedentes de familias que ya estaban aquí obtienen el graduado en un $92 \%$, en los 
alumnos de procedencia africana, es del $50 \%$ (en cuanto la población de origen marroquí es de un 70\%). Tenemos por delante muchos retos, y algunos urgentes.

Habrá tiempo por analizar, pero sorprende la edad de los que cometieron los atentados el pasado jueves. Algunos, ya nacidos en Cataluña. La mayoría de Ripoll, una ciudad pequeña del Pirineo de Girona. No procedían de la gran ciudad. Habrá que analizar caso por caso, y por diferentes agentes: educativos, sociales, religiosos, políticos. Culpidor el mensaje que circula por las redes de la educadora social de Ripoll y que conocía estos algunos de estos muchachos.

Tenemos que ser muy críticos con algunos mensajes aparecidos en las diferentes redes sociales, que simplemente fomentan el odio (castigado por nuestro código penal). Algunos grupos han llegado a convocar a manifestaciones en contra de dar becas escolares y ayudas sociales a personas de origen musulmán. Estamos en un estado de Derecho, en igualdad de obligaciones y de derechos para toda la ciudadanía, venga de donde venga, de que raza tenga o religión. El reto es grande, y tendremos que explicar bien las causas de este conflicto y no culpabilizar a toda una comunidad. El tema, como se ha dicho, es mucho más amplio. No sólo serán medidas policiales, que tendrá que haber, también otras si queremos vivir un mundo en paz y dignidad. Que se respeten todas las identidades, que son múltiples, y la gran diversidad en igualdad de condiciones.

Es preciso reconocer que la diversidad humana como normalidad y asumir la equidad y la justicia social como valores éticos irrenunciables. Los centros educativos pueden ser un buen espacio para llevar a cabo la educación intercultural como enfoque que afecta a todas las dimensiones del proceso educativo y a la sociedad en su conjunto. En estos momentos difíciles cabe gestionar la diversidad cultural, que no es una tarea fácil y es altamente compleja, de ella depende favorecer la convivencia intercultural o entorpecerla y alimentar un discurso del odio, como algunas manifestaciones de las redes sociales que se han descrito antes.

Como dice el profesor Besalú (Revista Convives, 2016, p. 30):

La educación intercultural tiene por objetivo el encuentro, el intercambio en pie de igualdad, conservando la propia identidad y, pues, sus destinatarios son todos los componentes de la sociedad; pretende conocer y modificar los distintos estereotipos y prejuicios, estimular el conocimiento y la valoración crítica de todas las culturas, promover actitudes, conductas y relaciones positivas y evitar la discriminación

$\cdots$ 
No confundir a las personas con el problema, ni el problema con el proceso: separar estos tres elementos nos permitirá ser sensibles y comprensivos con las personas, duros con el problema y equitativos en el proceso.

Recebido em Julho de 2017

Aprovado em Agosto de 2017

Publicado em Agosto de 2017 